\title{
BMJ Global Health Improving postpartum care delivery and uptake by implementing context- specific interventions in four countries in Africa: a realist evaluation of the Missed Opportunities in Maternal and Infant Health (MOMI) project
}

To cite: Djellouli N, Mann S, Nambiar B, et al. Improving postpartum care delivery and uptake by implementing context-specific interventions in four countries in Africa: a realist evaluation of the Missed Opportunities in Maternal and Infant Health (MOMI) project. BMJ Glob Health 2017;2:e000408. doi:10.1136/ bmjgh-2017-000408

Handling editor Seye Abimbola

- Additional material is published online only. To view please visit the journal online (http://dx.doi.org/10.1136/ bmjgh-2017-000408).

Received 16 May 2017 Revised 13 October 2017 Accepted 18 October 2017

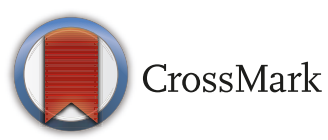

For numbered affiliations see end of article.

Correspondence to Dr Tim Colbourn; t.colbourn@ucl.ac.uk

\section{ABSTRACT}

Postpartum care (PPC) has remained relatively neglected in many interventions designed to improve maternal and neonatal health in sub-Saharan Africa. The Missed Opportunities in Maternal and Infant Health project developed and implemented a context-specific package of health system strengthening and demand generation in four African countries, aiming to improve access and quality of PPC. A realist evaluation was conducted to enable nuanced understanding of the influence of different contextual factors on both the implementation and impacts of the interventions. Mixed methods were used to collect data and test hypothesised context-mechanism-outcome configurations: 16 case studies (including interviews, observations, monitoring data on key healthcare processes and outcomes), monitoring data for all study health facilities and communities, document analysis and participatory evaluation workshops. After evaluation in individual countries, a cross-country analysis was conducted that led to the development of four middle-range theories. Community health workers (CHWs) were key assets in shifting demand for PPC by 'bridging' communities and facilities. Because they were chosen from the community they served, they gained trust from the community and an intrinsic sense of responsibility. Furthermore, if a critical mass of women seek postpartum healthcare as a result of the CHWs bridging function, a 'buzz' for change is created, leading eventually to the acceptability and perceived value of attending for PPC that outweighs the costs of attending the health facility. On the supply side, rigid vertical hierarchies and defined roles for health facility workers (HFWs) impede integration of maternal and infant health services. Additionally, HFWs fear being judged negatively which overrides the self-efficacy that could potentially be gained from PPC training. Instead the main driver of HFWs' motivation to provide comprehensive PPC is dependent on accountability systems for delivering PPC created by other programmes. The realist evaluation offers insights into some of the contextual factors that can be pivotal in enabling the community-level and service-level interventions to be effective.

\section{INTRODUCTION}

An estimated $29.4 \%$ of 0.29 million maternal deaths occur after delivery on the birth day, and a further $33.5 \%$ occur during the rest of the first month after birth; these percentages are $18.5 \%$ and $33.3 \%$ for the combined total of 5.5 million neonatal deaths and stillbirths. ${ }^{1}$ Yet, postpartum care (PPC) has remained relatively neglected in many interventions designed to improve maternal and neonatal health in sub-Saharan Africa. ${ }^{23}$ The Missed Opportunities in Maternal and Infant Health (MOMI) project ran from February 2011 until January 2016 in four African countries: Burkina Faso, Kenya, Malawi and Mozambique. The primary objective of the study was to improve maternal and newborn health through a focus on the postpartum period, adopting context-specific strategies to strengthen healthcare delivery and services at both facility and community level. $^{4}$ 


\section{Key questions}

\section{What is already known about this topic?}

- Postpartum care (PPC) has remained relatively neglected in many interventions designed to improve maternal and neonatal health in sub-Saharan Africa.

- Realist methods offer the opportunity for studying complexity of health systems and enhance understanding within the context of PPC, but are relatively uncharted territory methodologically for evaluating health system change in low-and-middle-income country settings.

\section{What are the new findings?}

- Influences on demand for PPC are related to two major mechanisms of social capital: bridging social capital enacted through the relationship between women and community health workers; and the development of bonding social capital or a 'buzz'.

- Healthcare workers' motivation to provide PPC is not driven by the training received but by accountability systems for delivering PPC in the wider policy context.

- Strict vertical hierarchies and defined roles for healthcare workers pose barriers to integration of maternal and infant services.

\section{Recommendations for policy}

- Community health worker interventions can create demand for care in the postpartum period.

- Need to tie quality indicators into routine reporting structures and supervision models to develop accountability for quality of care.

- Greater engagement and participation of the health systems leadership is necessary to bring about the changes needed for quality improvement and integration of maternal and infant services.

Using participatory methods in each study siteKaya district in Burkina Faso, Kwale county in Kenya, Ntchisi district in Malawi and Chiúta district in Mozambique-a package of postpartum interventions (table 1) was designed and developed, tailored to the implementation gaps identified from an initial situation analysis and country-specific participatory causal analysis workshops. ${ }^{5}$

MOMI's basic programme theory postulates that by strengthening PPC services, integrating maternal and child health services to optimise PPC delivery and increasing awareness of and demand for PPC services, processes for uptake and delivery of PPC would improve, thus enhancing the frequency and quality of PPC, leading eventually to improved maternal and infant outcomes. Evidence points to what is needed to influence the MOMI objectives of improving the uptake, and the frequency and quality of delivery of PPC but less is understood about for whom, how and under what circumstances interventions are likely to work. A tailored design approach was chosen to take account of context in maximising intervention effectiveness. A realist method of evaluation was undertaken in order to answer the following questions: which contexts (1) influence women's decisions to attend and benefit from the PPC that is on offer and (2) enable the frequency and quality of delivery of evidence-based PPC to be maximised; and which mechanisms lead to these outcomes. Country-specific findings as well as findings about the sustainability and replicability of the interventions were described elsewhere. ${ }^{6}$ i Here, the findings are presented across all four countries and the objective of our realist evaluation is to develop theory about how the interventions are thought to work, or not.

\section{METHODS}

\section{Evaluation design and data collection}

The overall evaluation of the MOMI project involved quantitative impact evaluation, measurement of implementation strength and analysis of programme theory using realist evaluation. ${ }^{6}$ In this paper, we focus on the realist evaluation. We undertook a realist evaluation ${ }^{78}$ so as to enable a more nuanced understanding of the influence of different contextual factors on both the implementation and impacts of the MOMI interventions, which are complex and operate at multiple levels: individual women and health workers, communities and health facilities. Realist evaluation was the principal evaluation methodology supported and informed by evaluation of implementation strength ${ }^{6}$ and simple before and after analyses of facility monitoring data (antenatal, delivery, postnatal and outpatient registers) and from community health workers' (CHWs') records. ${ }^{6}$ This gave an indication of progress and trends in uptake of care and outcomes (online supplementary appendix 1).

The first stage of the evaluation entailed developing the initial programme theories and the associated context $(\mathrm{C})$, mechanism $(\mathrm{M})$ and outcome $(\mathrm{O})$ configuration propositions (online supplementary appendix 2) shaped by five periods of data collection-the baseline policy analysis, ${ }^{3}$ the situation analysis, ${ }^{9}$ a causal analysis workshop with key stakeholders in each country setting, the development of interventions ${ }^{5}$ and the pilot evaluation data collected earlier in the phase of intervention implementation. The CMO configurations sought to unpack how, when, under what circumstances and for whom interventions were presumed to exert an effect. We aimed to further our understanding of how the health system strengthening interventions acted both at a social network and individual behaviour change level. We therefore used substantive theories-Pawson's theory of health system change, ${ }^{10}$ Michie's Behaviour Change Wheel $^{11}$ and social capital theory ${ }^{12}{ }^{13}$ - to frame the analyses of our primary data and to develop the testable programme theory.

The second stage of the evaluation involved testing and refining those initial programme theories through embedded multiple-case studies. ${ }^{14}$ A case was defined as a community unit including a health facility, whereby daily observations at the health facility and in the community

${ }^{\mathrm{i}}$ Parts of the results presented in this paper have already appeared in our full evaluation report (ref. 6), available here: http://icrh.org/ sites/default/files /MOMI\% 20Final\% 20Evaluation \% 20Report \% 20 \%28WP6\%20and\%20WP7\%29.pdf (accessed 10 Oct 2017). 
Table 1 Package of postpartum interventions in each study district

\begin{tabular}{ll}
\hline Study site & Selected interventions \\
\hline Burkina Faso-Kaya district & $\begin{array}{l}\text { 1. Enhance the delivery of immediate PPC in health facilities with focus on the detection and } \\
\text { management of postpartum haemorrhage and sepsis and PPFP }\end{array}$ \\
2. Integration of maternal and infant services in the postpartum period \\
$\begin{array}{l}\text { 3. Support mother and infant during the postpartum period through female CHWs conducting } \\
\text { home visits, providing individual counselling and group health education on PPC (including } \\
\text { FP) and by referring women to the health facility for scheduled PPC consultations and in } \\
\text { case of complications }\end{array}$
\end{tabular}

Kenya-Kwale county

1. Strengthening immediate PPC for mother and newborn by upgrading knowledge and skills of facility and CHWs on detection and management of common maternal and neonatal complications, promotion of exclusive breastfeeding, counselling and provision of FP and by providing postpartum home visits (conducted by $\mathrm{CHWs}$ )

2. Increase knowledge on and uptake of PPFP during the first year after delivery using the dialogue model at community and facility level

Malawi-Ntchisi district
$\begin{aligned} & \text { 1. Strengthening clinical management of PPC (using clinical mentorship and quality of care } \\ & \text { reviews) }\end{aligned}$
$\begin{aligned} & \text { 2. Increase utilisation of PPFP by providing counselling at health facility and community levels } \\ & \text { 3. Strengthening community PPC management through home visits conducted by CHWs and } \\ & \text { through the establishment and use of men's, women's and youth groups }\end{aligned}$
Mozambique-Chiúta district $\begin{aligned} & \text { 1. Upgrade mother and newborn postpartum risk assessment and management at facility and } \\ & \text { community level through the use of checklists }\end{aligned}$
$\begin{aligned} & \text { 2. Scale up access to and use of PPFP through making immediate postpartum intrauterine } \\ & \text { devices available } \\ & \text { 3. Improve access to and use of maternal PPC and services by integrating maternal PPC in } \\ & \text { child clinics and outreach activities }\end{aligned}$

All districts were assessed (to a greater or lesser extent dependent on the data available) as part of the realist evaluation reported in this paper.

CHWs, community health workers; PPFP, postpartum family planning; PPC, postpartum care.

were conducted over a 2-week period in conjunction with semistructured interviews with health facility workers (HFWs), CHWs affiliated with the health facility and postpartum women from the local community served by the facility. Four cases were purposively selected in each country study site to maximise geographical variation and various degrees of implementation effectiveness. A description of each case is available in online supplementary appendix 3 . Bespoke 'realist' data collection tools including interview topic guides and observation templates were designed to ensure that the theories were specifically tested. ${ }^{15}$ Cases were supplemented by semistructured interviews with policymakers and MOMI implementers. In total, across the 16 case studies, 52 postpartum women, $40 \mathrm{CHWs}, 46 \mathrm{HFWs}$ as well as 18 policymakers and 15 MOMI implementers were interviewed (see table 2). This wave of data collection further comprised monitoring data collected in each country throughout the implementation period, from the health facilities' registers and from CHWs' records. ${ }^{6}$ These indicators included cases of newborn and maternal complications and deaths from different causes, treatments given and interventions delivered, at both community and facility levels. Online supplementary appendix 1 contains the data collection tools used in each country.

A more detailed overview of the study design and data collection is available elsewhere. ${ }^{6} 16$

\section{Data analysis}

Qualitative and quantitative analyses were conducted. Interviews, observation logs, as well as MOMI reports and transcripts from participatory evaluation workshops held in each country after the case studies ${ }^{6}$ were analysed using thematic qualitative analysis. Codes were developed by the evaluation team and tested on a small sample of interviews (including interviews with women, HFWs and CHWs). Data were coded based on realist terms, that is, context, resource, reasoning and outcome. CMO configurations were extracted as units by identifying outcomes first and then, for each outcome, the associated mechanism followed by the associated context. ${ }^{17}$ Simultaneously, each CMO extracted was assigned a theme identified in the programme theories to be tested (online supplementary appendix 2). Grouping under themes simplified comparison between case studies, traceability and triangulation with other data sources. All sources of qualitative data were analysed in NVivo V.11 software, and memos recording emerging themes were shared between the evaluation team.

Meanwhile, graphs of the trends in indicators by month for each of the facilities in each country were produced. In addition to visually relating the trends observed for each facility in these graphs with the intervention timelines, the trends were compared and contrasted with the findings from the realist evaluation case studies. 


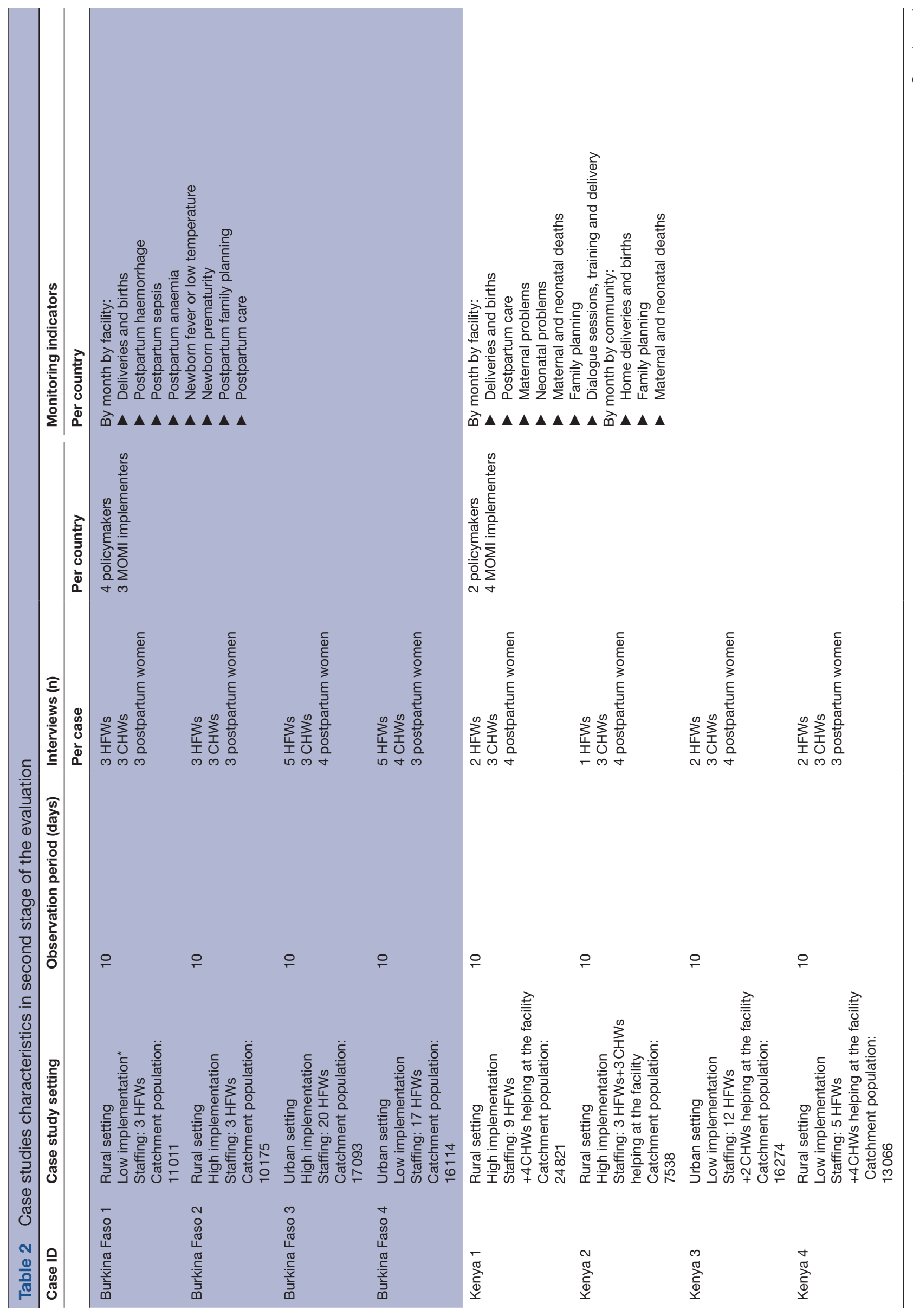




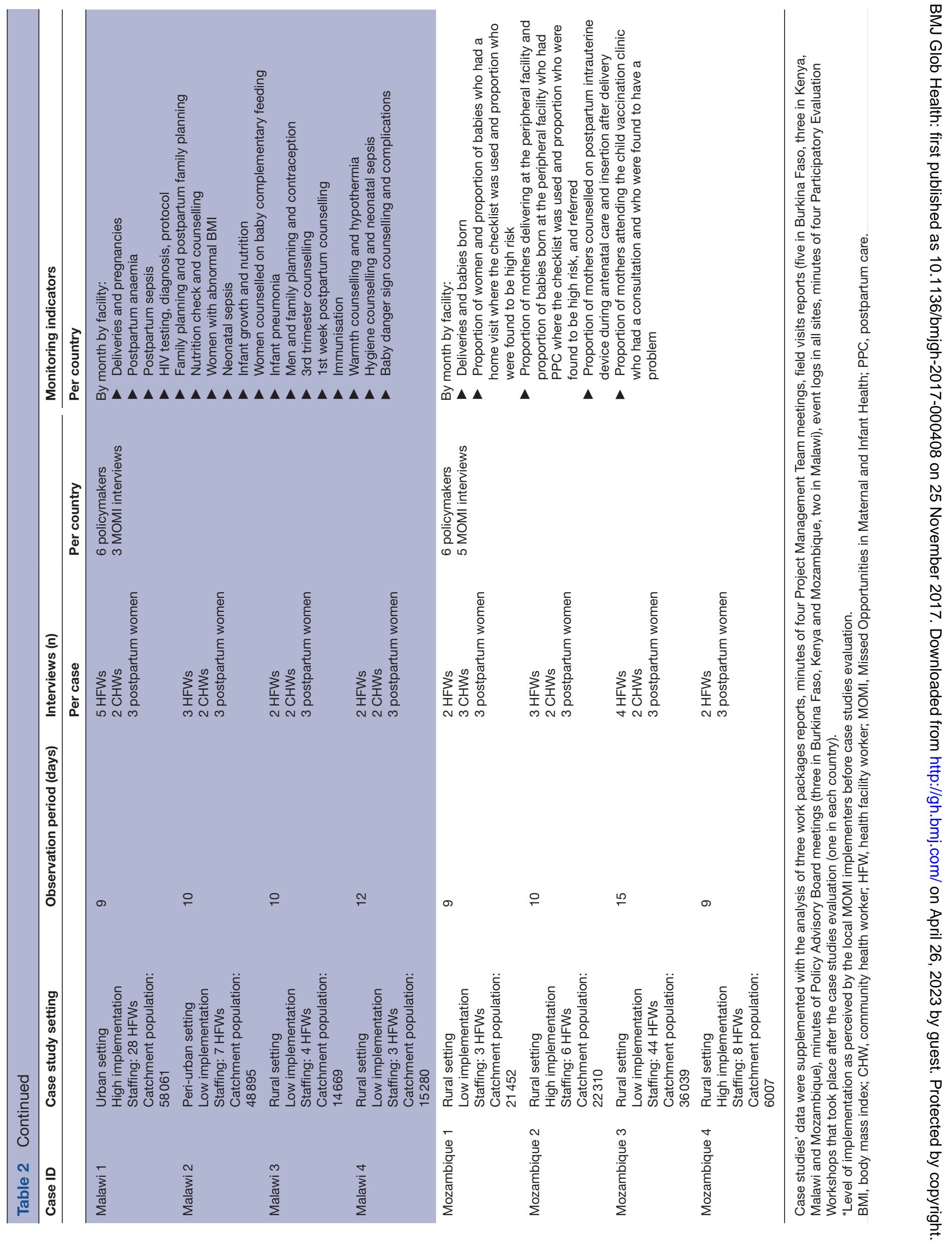


The themes emerging from the qualitative analysis were compared with the quantitative data in the same facilities to determine whether the programme theory was plausible given the data, and also to determine whether the data were plausible given the programme theory. The quantitative and qualitative data were analysed separately initially so as to avoid bias.

Findings were discussed and triangulated between the core evaluation team from University College London in weekly meetings during the final fourmonths of the evaluation (October 2015-January 2016). Additionally, the analysis was conducted in very close consultation with the research teams in-country who collected the data to cross-check interpretations and emerging findings.

\section{Developing middle-range theories}

Middle-range theories (MRTs) aim to find concepts with a sufficient level of abstraction from the empirical underlying mechanisms and context so as to provide a cumulative, logical explanation of the changes being observed. ${ }^{18}$ After refining the initial programme theories through data analysis and application of findings to initial CMO configurations at the individual country level, the MRTs were developed. Through a process of testing between countries and cross-referencing, the team were able to abstract the findings further and identify common propositions that were generalisable across different contexts to synthesise the four MRTs presented. Identification of the facilitating or hindering contexts and their influence on realisation of the outcomes within the different countries enabled the MRTs to be reached. These were then checked through negotiation and interpretation of the data with members of the evaluation team and further checked and finalised with the rest of the MOMI consortium during the final programme management team meeting and with stakeholders during the MOMI dissemination conference (January 2016, MombasaKenya). Written informed consent was obtained from all participants interviewed during the evaluation.

\section{RESULTS}

We present here four broad MRTs—named 'bridging theory', 'buzz theory', 'motivation by accountabilities' and 'together is stronger'-elicited from the evaluation data which underpinned whether or not the interventions implemented had an impact at the point of service delivery despite some variation in intervention choice, design and delivery across settings and differences within the contexts and systems within which they were implemented.

\section{Bridging theory}

CHWs in all study sites were to conduct home visits during the postpartum period to engage with mothers about the importance of PPC, the identification of postpartum and postnatal danger signs and the need to attend the health facility for follow-up visits for both mother and infant. These home visits were complemented by community sensitisation events on PPC. In practice, the range of roles undertaken, the degree to which the CHWs were linked to the formal health sector and the way they were incentivised by the system and/or MOMI project varied in each of the four settings.

Yet, trust from the community was identified as a crucial element for the success of community interventions. CHWs gained the trust of the community because CHWs were perceived to belong to and were selected by the community they served. CHWs also gained trust and role identity from their CHW status within the community and influenced the uptake of PPC in the community. This gave CHWs a strong intrinsic sense of responsibility to their communities and contributed to the community relying on them, which contributed to their motivation to provide an effective bridging function.

At first we were facing some difficulties. But over time trust was established. Because we were appointed to be CHWs, the population knows about us, plus since we are from the same community we benefit from some credibility. (CHW-Burkina Faso)

These are my people and I want the best for them. If I don't do my work they'll perish. (CHW-Kenya)

My relationship with the community is good because I am the son of the area and they already know and are used to me since I save many lives. (CHW-Mozambique)

In order for CHWs to further fuel their motivation and to effectively forge links with the formal sector, their recognition here was also important and was established through different mechanisms such as training, close supervision and incentives-all of which built a sense of belonging with the health facility. Local CHWs already hold the trust of the community and making the health facility links more visible reinforces this trust in the system and establishes connectivity. Visible signs of connection to the formal sector such as uniforms and MOMI bicycles, and the use of pictorial checklists appeared to work through this mechanism and be motivating for the CHWs.

There is some women who want to come but maybe they are scared of the HFWs. But they tell themselves that if there is [a CHW] who works with health workers, who collaborates with health workers, if I am accompanied by her, all my problems, my worries would maybe diminish with her assistance. (HFW-Burkina Faso)

We are like the bridge linking the community to the health facility. If we are not there, they will not have any one sensitizing them on health matters. We act like ambassadors passing information back and forth. (CHW-Kenya)

CHWs have an important role in the community, if the mother has problems, it is them that are the first point of call. They act as a liaison between the community and health facility. (HFW-Mozambique)

Figure 1 shows the links between the different CMO configurations and how trust from the community and CHWs' motivation are prerequisites for an effective 


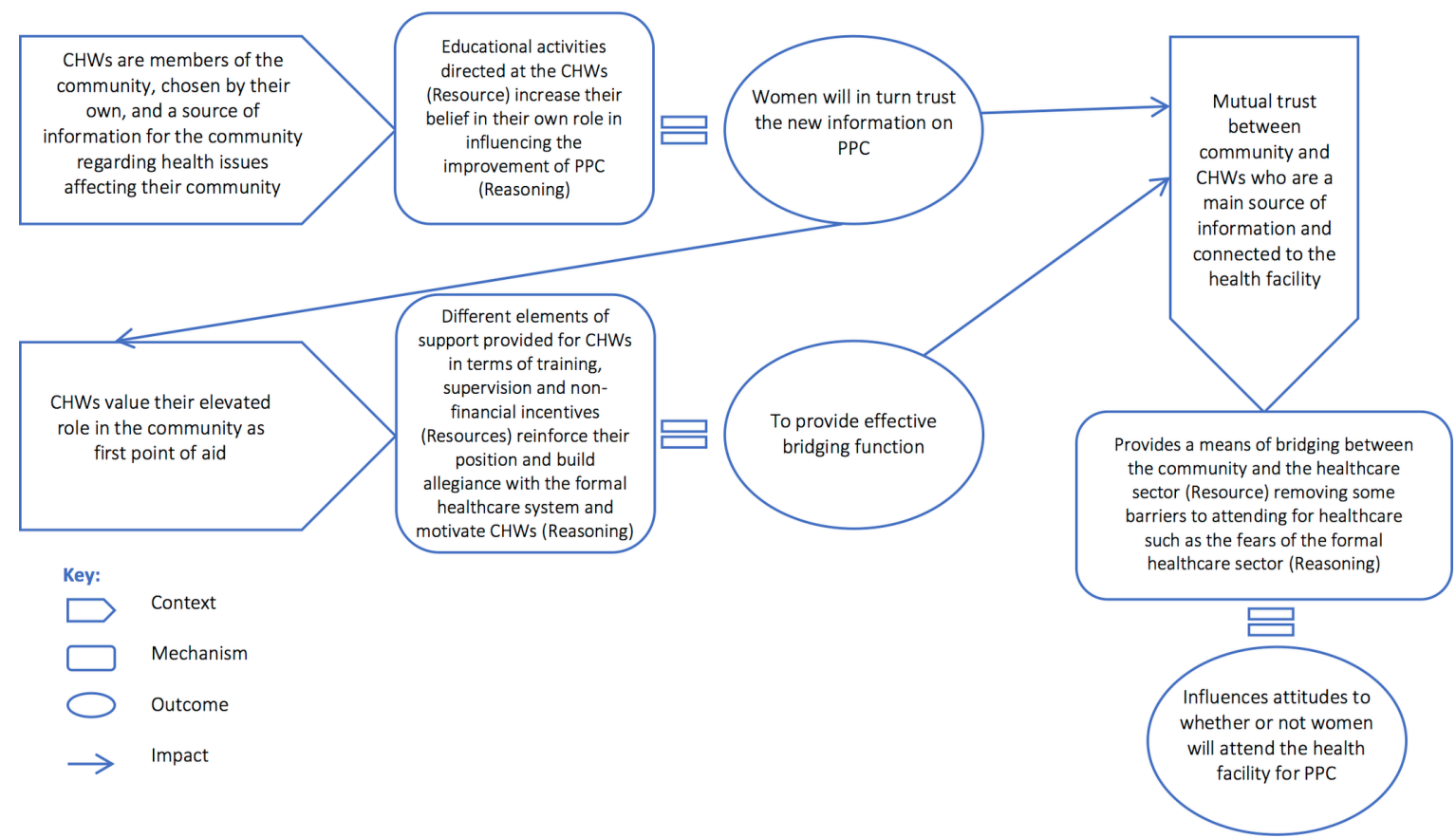

Figure 1 Context-mechanism-outcome configurations for the bridging theory. CHW, community health worker; PPC, postpartum care.

bridging function that can lead to more women attending the health facility. In Burkina Faso, this bridging process was observed to be a more important driver of behaviour change in rural areas than in urban communities where the relationships of the community with CHWs and in particular satisfaction of the CHWs appear to be different. This is mainly because female CHWs are able to find more profitable activities in urban settings.

MOMI, it's efforts that are not rewarded! This is the bag that they gave us to do the work, in which there is my blouse, but I preferred to leave it and invest myself in my business in order to satisfy my needs and those of my children. (...) The 2000 francs [compensation] can't cover the expenses so I decided to slow down the MOMI activities and focus on my profitable activities. (CHW-Burkina Faso)

Yet, the retention for CHWs remained high even in urban settings as CHWs were motivated throughout the project through the mechanisms discussed. In Malawi, it was not possible to comment on the demand generation for PPC through the CHWs' bridging function due to the lack of implementation of the community interventions.

\section{Buzz theory}

Our refined theory through the MOMI evaluation suggests that influences on demand for PPC occurring at a community level are related to two major mechanisms of social capital (the relationships between people within the community) - (1) bridging social capital enacted through the relationship of women with the community health workers and (2) the development of bonding social capital or a 'buzz', as illustrated in figure 2. These factors appear to be leading initially to cognitive changes in the form of building on existing and new trust relationships, followed by behavioural changes, resulting in an increase in healthcare demand.

If community-level interventions lead to postpartum healthcare seeking for a critical mass of women, a 'buzz' for change is created. Reinforced by social cohesion and local dialogue, norms shift and appear to create a critical tipping point leading to a social movement that holds a collective belief in the acceptability of and perceived value of attending for PPC that outweighs the costs of attending the health facility.

In Burkina Faso, all CHWs and some postpartum women explained that women adopt the behaviour of other women. Therefore, the women that first attended PPC consultations-following CHWs' recommendations-were satisfied and shared their experience with other women in their communities who then decided to also attend the health facility for PPC.

These women who refused at first are now those who adhere the most because they saw the others who adhered and were well so they decided to adhere as well. (...) Those that refused at the beginning we didn't have to go sensitise them. It's the fact they saw the positive change in the life of the other women that made them decide to adhere too. (CHW-Burkina Faso)

It's when the first women started to do it and we knew there was nothing bad to it but on the contrary it was to help us, that we started to get used to it little by little. (Postpartum woman-Burkina Faso)

As a result, MOMI community interventions led by CHWs, reinforced by a 'buzz' in the community, did increase demand for PPC, which was also supported by quantitative data (online supplementary appendix 4, 


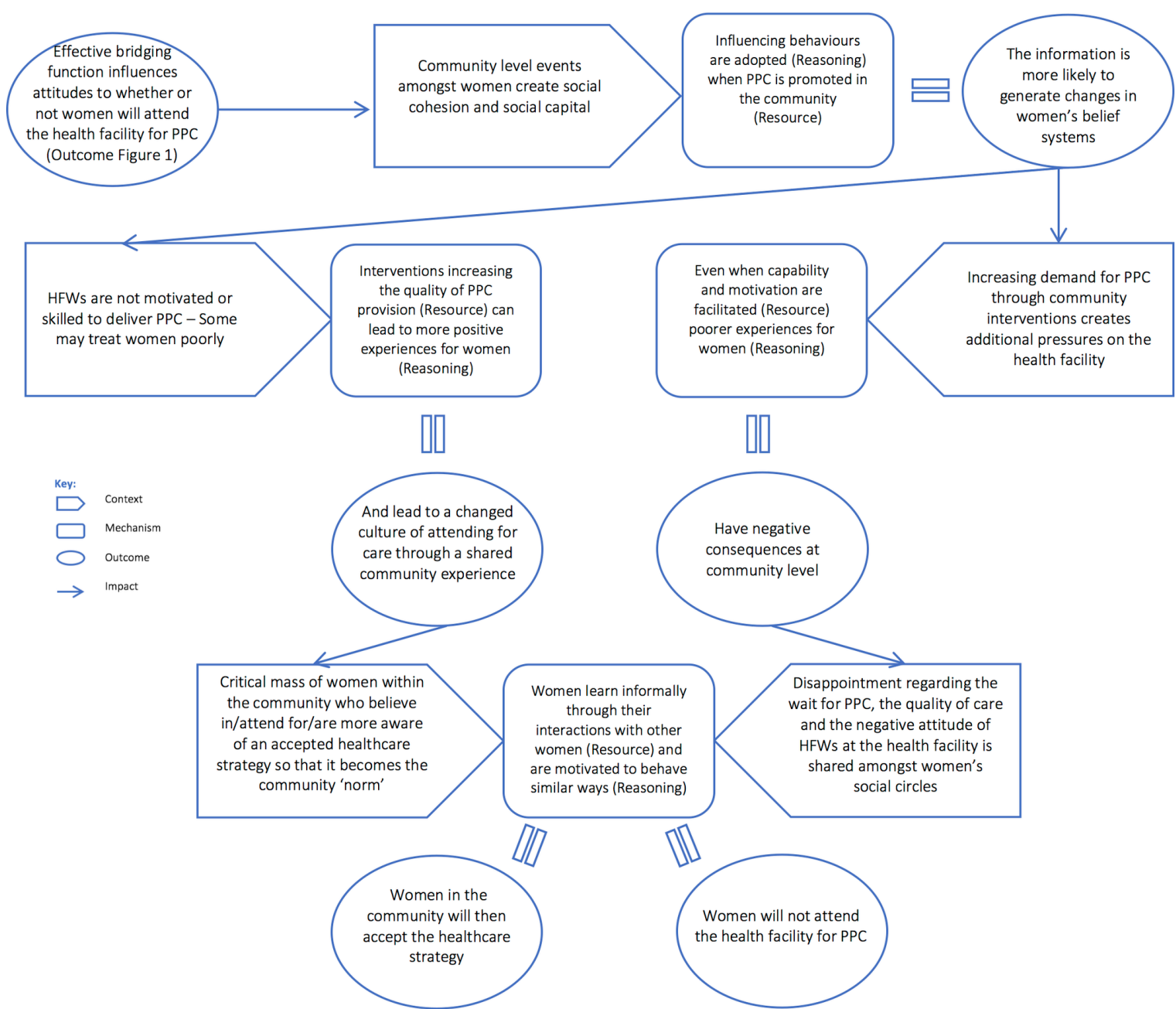

Figure 2 Context-mechanism-outcome configurations for the buzz theory. HFW, health facility worker; PPC, postpartum care.

figure A4.1). Increases post-March 2014 (online supplementary appendix 4, figure A4.1) could also be due to the pay-for-performance scheme piloted in the district.

In Mozambique, the CHWs were instrumental in encouraging some women from the community to attend PPC, although penetration into the community was not sufficient to generate the 'buzz' that was captured in Burkina Faso. This was thought to be due to geographical barriers to the community reaching the health facility and an insufficient number of CHWs to achieve good coverage of scattered and isolated communities. This precluded the development of the required social capital 'threshold' required to influence norms of behaviour and therefore achieve a critical mass to reinforce the sustainability of the CHW intervention. In Kenya, this mechanism for generating demand was not captured in the data while Malawi did not implement its community intervention.

Furthermore, it should be noted that to generate a positive 'buzz' about PPC some contextual and implementation factors are crucial, in all settings: the active bridging function of CHWs; the support from community leaders; and especially a good PPC experience at the health facility once women make the decision to attend. Otherwise a 'buzz' might be generated to not attend the health facility for PPC (figure 2).

You know if one experiences badly, they will in turn go and tell others of what they experienced. (CHW-Kenya)

A member of a MOMI community group reported than when she went for PPC check-up she had expectations that the nurse would check her but the nurse just checked the baby and nothing was done to the baby. (...) When the MOMI researchers followed up on this issue, they were told that the community is disappointed with the quality of services and the negative attitude of the health workers. (Field observations-Malawi)

\section{Motivation by accountabilities}

In all four countries, HFWs were found to be constrained in their daily duties by many factors, including lack of training, human and material resources; significant workload in maternal and child healthcare; high levels of bureaucracy; and isolation for those placed in rural sites. Furthermore, due to fixed hierarchies, innovation and initiative-taking among HFWs are often not rewarded. 

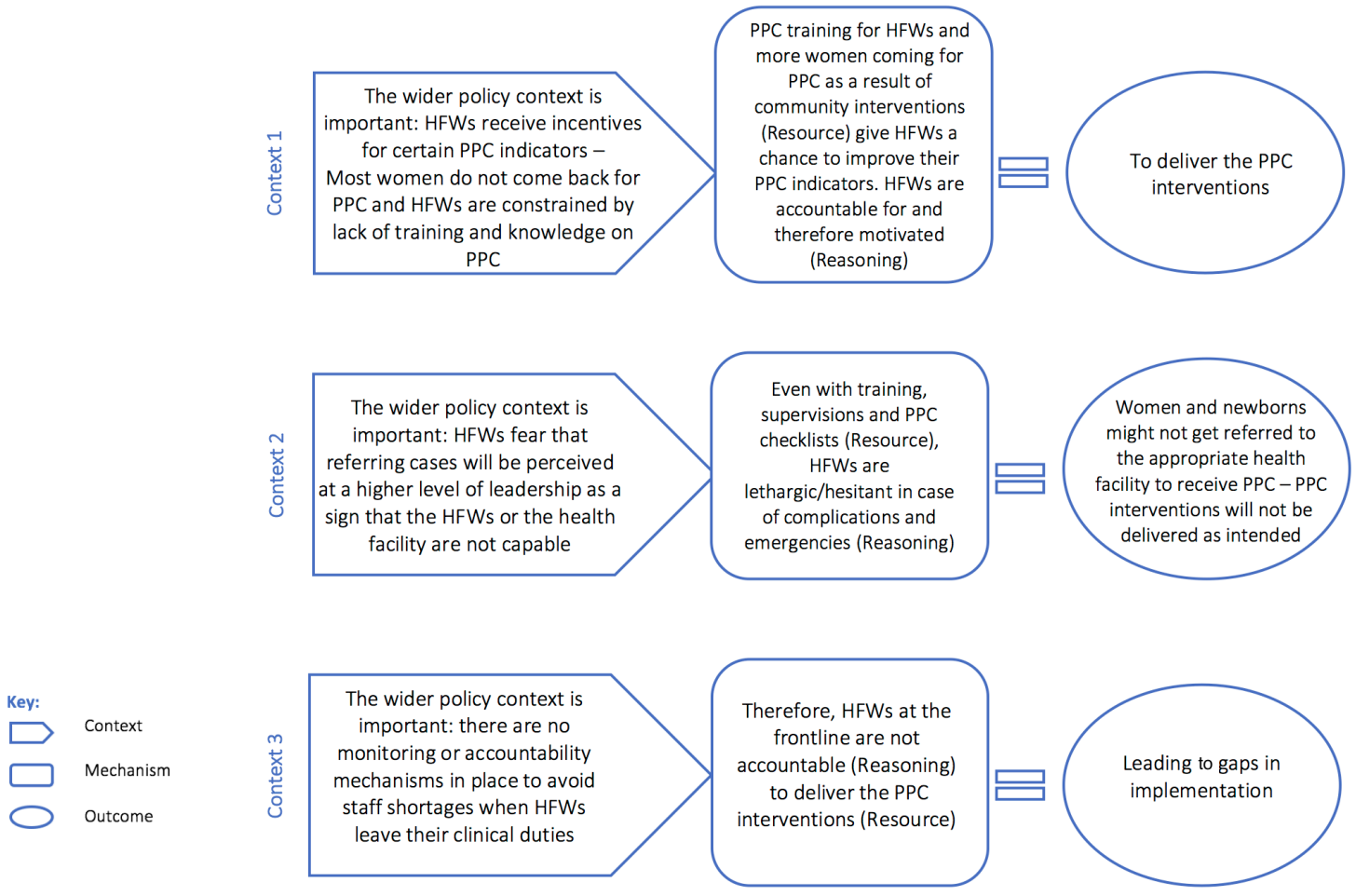

Figure 3 Context-mechanism-outcome configurations for the motivation by accountability theory. HFW, health facility worker; PPC, postpartum care.

Performance of HFWs and the health facility overall is judged and rewarded by reported activity, rather than health system responsiveness or quality of care. HFWs fear being judged negatively which overrides the self-efficacy that could potentially be gained from PPC training. Although the MOMI training was very well received in all countries, it was not sufficient to increase the provision of comprehensive PPC. Rather, our findings suggest that HFWs' motivation to provide PPC is not related to the training received or other MOMI interventions but to accountability systems for delivering PPC in the wider policy context.

In Burkina Faso, a pay-for-performance system was being piloted in Kaya district from March 2014 onwards that bought several national indicators including some relevant to PPC (PPC consultations at days 6 and 42; postpartum family planning (PPFP)). The system was in synergy with the MOMI interventions that increased women's attendance for PPC and supported an increase in activity, motivating HFWs to deliver PPC. Consultations at days 6 and 42 and offer of PPFP was increased as a result of introducing the MOMI interventions but this change was augmented with the introduction of the pay-for-performance pilot (online supplementary appendix 4, figures A4.1 and A4.2).

Despite being able to demonstrate this increase in activity, it is not clear that this also resulted in improved quality. However, it did motivate HFWs to provide PPC.

Well [PPC] was a care that was neglected before, we neglected postpartum women before. But now, with MOMI and pay-for-performance, this care is remunerated. So it means that currently there is enthusiasm. (HFW-Burkina Faso)

In Mozambique, the number of referrals to higher facilities was monitored and seemed to be in line with the MOMI checklists implemented whereby critical postpartum complications cases were to be referred to a higher-level facility. However, HFWs were demotivated to send women and/or infants to the referral facility—even when the complication was correctly diagnosed-since they feared that this would be perceived at district or provincial level as a sign that the health facility and its workers were incapable. HFWs refused to comment on this in recorded interviews although it was observed on several instances that they were apathetic in emergency situations, delaying transfer to another facility or avoiding it altogether, leading sometimes to the death of the mother and/or infant.

Serious cases are not treated as if they were urgent, and they keep on delaying transport for the patients to the referral hospital, and patients arrive at the hospital in critical condition. (Field observations-Mozambique)

[HFWs] are worried that referring will be interpreted as a sign of maybe being incapable. (MOMI implementerMozambique)

However, it was also observed that some HFWs did refer complication cases without reporting it to keep the referral numbers low (online supplementary appendix 4, figure A4.3). This assumption was later confirmed during the participatory evaluation workshop. 


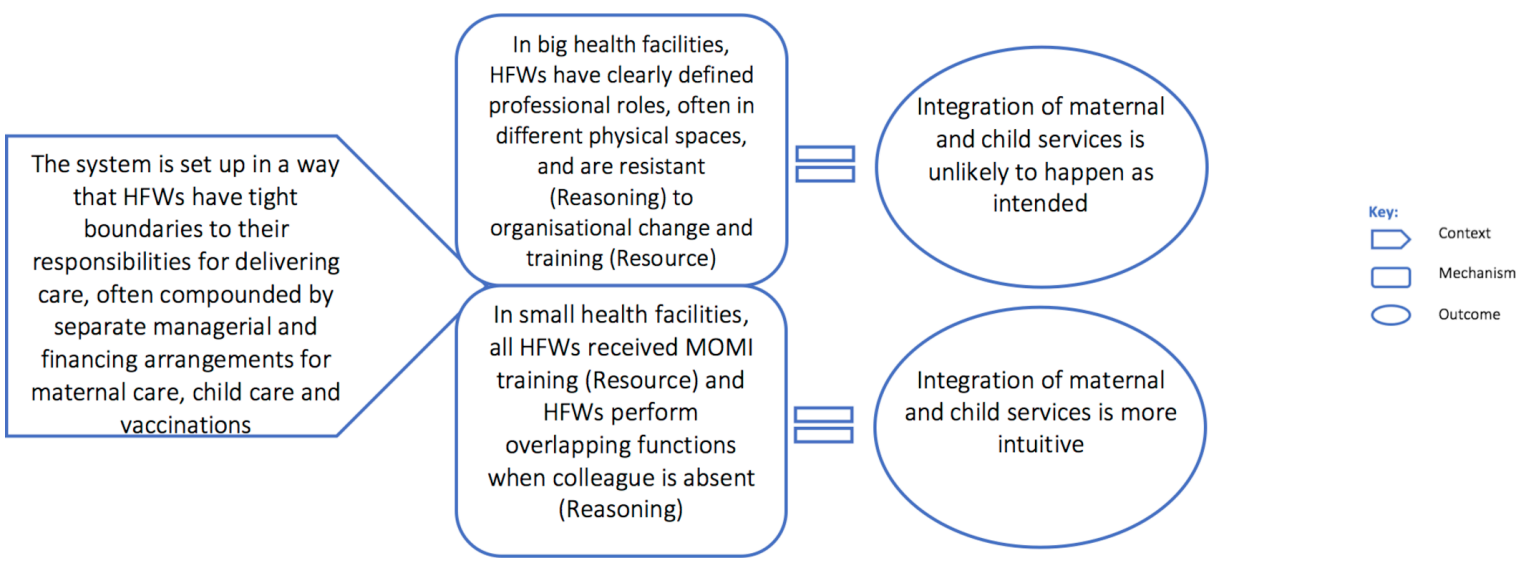

Figure 4 Context-mechanism-outcome configurations for the together is stronger theory. HFW, health facility worker; MOMI, Missed Opportunities in Maternal and Infant Health.

In Malawi, there were no monitoring or accountability mechanisms in place to prevent staff shortages resulting from HFWs leaving their clinical duties to attend personal business or to undertake paid activities with non-governmental organisations and other stakeholders. Staff shortages in addition to a large workload for maternal and child services result in the remaining staff being overwhelmed and demotivated to implement and deliver PPC interventions.

There are no consequences to whatever we [HFWs] do. You can neglect a patient, you can do whatever, but there are no consequences. (Participatory Evaluation Workshop participant-Malawi)

For instance MOMI, the way I heard about it, is the idea of giving PPC to a woman at one week postnatal check-up then, six weeks then three months was good. (...) But now it stopped due to lack of staff and everything ended there and then. (HFW-Malawi)

Figure 3 illustrates which mechanisms are triggered depending on the different wider policy contexts.

\section{Together is stronger}

Integration of services was planned in three out of four of the sites in order to use the infant vaccination visit as an opportunity to provide maternal care and family planning, as well as to capture postpartum women attending the health facility for other services. The aim was to deliver these service functions in one setting reducing fragmentation of the patient journey and potentially enhancing the number of services that the women were able to access contributing to the increasing the frequency of delivery' aim of MOMI. In practice, explicit integration of different elements of the interventions may have been poorly conceptualised at the outset, and consequently, the planning and execution of the integration of services did not initially involve the structural and organisational reconfigurations that were needed.

When the health facility staff perceive the problem, the implementation of the suggestions, it works. But when the staff doesn't perceive the problem the same way than us, we often have to explain and re-explain. (MOMI implementer-Burkina Faso)

This limited the extent to which integrated delivery could really be provided since the services are traditionally provided through different systems, financing arrangements and in physically different places. In our evaluation, we were not able to study in more depth the contexts within which integration worked better or less well, except to develop theory about the relationship between size, complexity and level of resources the health facilities had-small, medium and larger facilities-and their prospects for delivering integrated care in the climate of limited resources. In general, it was found that where integration had been attempted, the staff in the better-resourced health facilities (six or more HFWs) were observed to have more clearly defined professional roles with little overlap between maternal and infant healthcare and therefore the combined provision of the services was less easily achieved.

We noted that there is an interpretation that the services have to be broken down, where for example, the $\mathrm{MCH}$ nurse says she cannot vaccinate babies because it is the work of her colleague. Before she did it when there was no technician for the area. (Field observations-Mozambique)

Although the study sites planned integration as one of their interventions, this was not observed explicitly in any of the sites. Nonetheless given that a function of integration is to provide PPC to both mother and child, we were able to observe the different aspects of context that motivated co-delivery. In a smaller facility (three or less HFWs), individual HFWs were often co-located, knew about each other's roles and expected to perform overlapping functions to account for absences. The opportunity for maternal care created by infant vaccination was perceived and performed more intuitively by HFWs in smaller rather than larger facilities. In essence, the smaller facilities were implicitly integrated just through the demand for greater diversification of roles and a need 
for flexibility. They were therefore more likely to offer opportunistically both maternal and infant care rather than depend on proactive attendance at two different settings (figure 4 ).

\section{DISCUSSION}

Realist methods offer the opportunity for studying complexity and enhancing understanding of context, but are relatively uncharted territory methodologically for evaluating health system change in low-and-middle-income country (LMIC) settings. ${ }^{19}$ In addition, the principle of MOMI was to implement a complex and diffuse set of health system strengthening interventions to improve PPC. Others have already recognised the challenge of understanding the interrelationships between individual, interpersonal, organisational and institutional level effects, and moving up and down the levels of abstraction to reach a MRT that is both generalisable but also relevant to the design of future interventions. ${ }^{20}$ There were similarities and differences to the different countries, and the theories reflected a level of abstraction that capitalised on commonalities while aiming not to be so abstract that they offered no useful new insights.

In many African countries, demand-side interventions have been effective in shifting demand for care in the antenatal period. ${ }^{21}$ The MOMI project demonstrated the potential for replication in the postpartum period. CHWs were a key asset as the bridge between community and health sector. Two components were crucial to effective bridging: trust from the community and CHWs' intrinsic motivation. Choosing CHWs from the same community they served established trust with the community and gave CHWs an intrinsic sense of responsibility, motivating them to conduct their activities even when facing barriers. As other studies have shown in different settings, ${ }^{22-24}$ CHWs' motivation is intrinsic but also driven by the elevated status gained in the community. Their status is reinforced by the support provided in terms of infrastructure, training, supervisions, incentives and connectedness with the formal health sector. Therefore, broadening the scope of their work can provide a key resource for raising the profile of PPC and facilitating access to routine PPC.

This evaluation however indicates that the CHWs' bridging function is interconnected with social capital mechanisms. Little is known, especially for sub-Saharan African countries, on the mechanisms through which social capital influence women's access to maternal care. ${ }^{25} 26$ Our MRT, in keeping with substantive theories of bonding and bridging social capital, ${ }^{27}$ suggests that forces of social cohesion have a powerful influence on healthcare behaviours and sheds some light on such mechanisms: if the CHWs' intervention leads to postpartum healthcare seeking for a critical mass of women, a 'buzz' for change is created through existing social cohesion mechanisms. This 'buzz' was observed in Burkina Faso, leading to further speculation that if the buzz theory gained enough ground it would become the overriding determinant of behaviour and the CHWs' bridging function might eventually not be needed. On the other hand, if the demand for PPC is not met by appropriate healthcare supply, a negative buzz might be generated to not attend the health facility for PPC as noted in Malawi.

Our third theory highlights that HFWs' motivation is mostly extrinsic due to the way the norms and values of the system operate where fixed hierarchies are valued more highly and initiative-taking among HFWs is not rewarded. There is a fear at all levels of being judged negatively and of sanctions ${ }^{28}$ that overrides the self-efficacy that has the potential to be gained through training, coaching support or supervision. ${ }^{29}$ Therefore, training and supervision on PPC-although well received-were not drivers to increase the provision of comprehensive PPC. A crucial determinant was instead contextual: accountability systems for delivering PPC. In settings where the accountability system worked in synergy with the MOMI project, HFWs were motivated to deliver the PPC interventions. In settings where it was not in synergy or absent, HFWs had no extrinsic incentives to implement the MOMI interventions. Investigating the reporting structure in place is thus necessary as it could be either an important facilitating or inhibiting implementation factor. However, there is no evidence from MOMI findings to suggest that increased activity through accountability systems necessarily translates into improved quality of care. Without tying quality indicators into routine reporting structures and supervision models-therefore developing accountability for quality ${ }^{30}$ - it would be hard to achieve improvements in quality or indeed to measure them for an intervention that involves working within the existing constraints. Our findings are in agreement with an ongoing systematic review of interventions to improve health worker performance, which finds training alone is insufficient and that accountability mechanisms are also needed (personal communication, Alexander K. Rowe, Centres for Disease Control and Prevention, 2017).

Studies on the effectiveness of integrating services in LMIC are limited. ${ }^{31} 32$ The results of our efforts towards integration are also limited due to defined job roles and strict vertical hierarchies, whereby challenging superiors is inconceivable. Integration was poorly understood by HFWs and was more intuitively performed in small facilities due to limited human resources. In larger facilities, HFWs were resistant to task-share and take on what was perceived to be other colleagues' responsibility. Integration of service delivery requires organisational and management integration and reorganisation of care practices as well as training. Greater engagement and participation of the health systems leadership is necessary to bring about these changes and more time should be devoted to conceptualising integration before implementation. 
Thus, a whole systems approach (including community bridging actors) to improvement needs to be taken into consideration rather than an intervention-focused approach. ${ }^{33}$

Some women interviewed in our study settings would not answer freely some of the questions (eg, around family planning themes, decision-making dynamics), making it difficult to elicit mechanisms of change. Some difficulties also arose with a few HFWs refusing to be recorded for fear of being reported (even though confidentiality was assured) to their supervisors. Furthermore, HFWs are not empowered to question leadership and hierarchy and most were reluctant to do so during interviews, limiting our ability to test programme theories around leadership. Finally, a certain degree of implementation strength is required for realist evaluation to test programme theories and to draw links between CMO configurations. In Malawi, for example, where implementation was delayed and limited, only a few programme theories could be tested.

\section{CONCLUSIONS}

While countries are making substantial progress in maternal and newborn health, further improvements can be achieved by implementing innovative interventions in the postpartum period. Strengthening health systems, integrating service delivery for the postpartum period and promoting demand for PPC through community interventions offer potential for success; and realist evaluation can help investigate how, where, for whom and in what circumstances such successful interventions work.

\section{Author affiliations}

${ }^{1}$ UCL Institute for Global Health, London, UK

${ }^{2}$ Faculdade de Medicina, Universidade do Porto, Porto, Portugal

${ }^{3}$ Institutde Recherche en Sciences de la Santé (IRSS), Ouagadougou, Burkina Faso

${ }^{4}$ International Centre for Reproductive Health—Kenya (ICRHK), Mombasa, Kenya

${ }^{5}$ Parent and Child Health Initiative (PACHI), Lilongwe, Malawi

${ }^{6}$ International Centre for Reproductive Health—Mozambique (ICRHM), Maputo, Mozambique

${ }^{7}$ Faculdade de Medicina, Universidade Eduardo Mondlane (UEM), Maputo,

Mozambique

${ }^{8}$ Chiúta District Health Department, Tete, Mozambique

${ }^{9}$ International Centre for Reproductive Health of the Ghent University (UG-ICRH), Ghent, Belgium

Acknowledgements The authors thank all the participants for sharing their insights and experiences with the research team, as well as the various health departments and facilities involved in the implementation of the MOMI interventions in each country.

Contributors ND was one of the evaluators; she coordinated and supervised data collection in all sites, designed the data analysis plan, led the qualitative data analysis and drafted the manuscript. SM was one of the evaluators; she designed the realist evaluation protocol, contributed to the qualitative data analysis and helped revise the paper, contributing intellectual content. BN was one of the evaluators; he contributed to evaluation design and analysis, and helped revise the paper, contributing intellectual content. PM coordinated the participatory evaluation workshops in all sites, contributed to the qualitative data analysis as part of the evaluation team and commented on earlier drafts. DM and $\mathrm{HB}$ contributed to the qualitative data analysis as part of the evaluation team. FYB, WMEY, CY and SB conducted the qualitative fieldwork in Burkina Faso. HT was involved in the implementation of the MOMl interventions in Burkina Faso, collected quantitative data and commented on earlier drafts. AC was involved in the implementation of the MOMI interventions in Burkina Faso and collected quantitative data. SK coordinated the MOMI research at the Burkina Faso study site and the quantitative monitoring at all four sites. VM and OKM conducted the qualitative fieldwork in Kenya. VM was also involved in the implementation of the MOMI interventions in Kenya. El was involved in the implementation of the MOMI interventions in Kenya and contributed to the interpretation of the results during stakeholder workshops. PG coordinated the MOMI research at the Kenya study site and contributed to the interpretation of the results during stakeholder workshops. ZD and AK conducted the qualitative fieldwork in Malawi. ZD was also involved in the implementation of the MOMI interventions in Malawi. CM coordinated the MOMI research at the Malawi study site and contributed to the interpretation of the results during stakeholder workshops. JT, MGC and MM conducted the qualitative fieldwork in Mozambique. MM was also involved in the implementation of the MOMI interventions in Mozambique. SG coordinated the MOMI research at the Mozambique study site and helped revising the paper. NBO coordinated the MOMI research at the Mozambique study site and commented on drafts of the paper. SF was involved in the implementation of the MOMI interventions in Mozambique and contributed to the interpretation of the results during stakeholder workshops. EO coordinated the MOMI consortium along with ED and contributed to the interpretation of the results during stakeholder workshops. ED was the overall MOMI consortium scientific coordinator and helped revising the paper. TC was one of the evaluators; he designed and led the quantitative component of the evaluation, contributed to evaluation design and analysis, and helped revise the paper, contributing intellectual content. All authors read and approved the final version of the manuscript.

Funding This paper is an output from the project 'Missed Opportunities in Maternal and Infant health: reducing maternal and newborn mortality and morbidity in the year after childbirth through combined facility and community-based interventions-MOMI'. The research leading to these results has received funding from the European Community's Seventh Framework Programme (FP7/2007-2013) under grant agreement no. 265448. MOMl is a collaborative project between International Centre for Reproductive Health—Ghent University (Belgium), Institute de Recherche en Sciences de la Santé (Burkina Faso), International Centre for Reproductive Health (Kenya), Parent and Child Health Initiative Trust (Malawi), International Centre for Reproductive Health Mozambique, Eduardo Mondlane University (Mozambique), Medical Faculty of the University of Porto (Portugal) and University College London (UK).

\section{Competing interests None declared.}

Ethics approval Ethics Committee for Research in Health of Burkina Faso; the University of Nairobi/Kenyatta National Hospital ethical review committee (Kenya); the National Health Sciences Research Council (Malawi); the National Health Bioethics Council (Mozambique).

Provenance and peer review Not commissioned; externally peer reviewed.

Open Access This is an Open Access article distributed in accordance with the Creative Commons Attribution Non Commercial (CC BY-NC 4.0) license, which permits others to distribute, remix, adapt, build upon this work non-commercially, and license their derivative works on different terms, provided the original work is properly cited and the use is non-commercial. See: http://creativecommons.org/ licenses/by-nc/4.0/

(c) Article author(s) (or their employer(s) unless otherwise stated in the text of the article) 2017. All rights reserved. No commercial use is permitted unless otherwise expressly granted.

\section{REFERENCES}

1. Lawn JE, Blencowe H, Oza S, et al. Every Newborn: progress, priorities, and potential beyond survival. Lancet 2014;384:189-205.

2. Sacks E, Langlois ÉV. Postnatal care: increasing coverage, equity, and quality. Lancet Glob Health 2016;4:e442-3.

3. Mann S. Cross-country analysis of maternal, newborn and child health policies in BurkinaFaso, Kenya, Malawi and Mozambique. London: University College London, 2013.

4. Missed opportunities in maternal and infant health. Missed opportunities in maternal and infant health (Internet). http://www. momiproject.eu/.

5. Duysburgh E, Kerstens B, Kouanda S, et al. Opportunities to improve postpartum care for mothers and infants: design of contextspecific packages of postpartum interventions in rural districts in four sub-Saharan African countries. BMC Pregnancy Childbirth 2015;15:131. 
6. Djellouli N, Mann S, Nambiar B, et al. Final evaluation of the MOMI project in Burkina Faso, Kenya, Malawi and Mozambique. London, Porto: University College London, University of Porto, 2016.

7. Pawson R, Tilley N. Realistic evaluation. London: Sage Publications, 1997.

8. Pawson R. The science of evaluation. A realist manifesto. London: Sage publications, 2013.

9. Barros H, Lopes S. Cross-country situation analysis of maternal and newborn care in Burkina Faso, Kenya, Malawi and Mozambique. Porto: University of Porto, 2013.

10. Pawson R, Greenhalgh J, Brennan C, et al. Do reviews of healthcare interventions teach us how to improve healthcare systems? Soc Sci Med 2014:114:129-37.

11. Michie S, van Stralen MM, West R. The behaviour change wheel: a new method for characterising and designing behaviour change interventions. Implement Sci 2011;6:42.

12. Bourdieu P. Le capital social. Actes Rech Sci Soc 1980;31:2-3.

13. Kreuter M, Lezin N. Social capital theory: implications for community-based health promotion. In: DiClemente R, Crosby R, Kegler M, eds. Emerging theories in health promotion practice and research: strategies for improving public health. San Francisco, CA: John Wiley \& Sons, 2002.

14. Yin RK. Case study research design and methods. Thousand Oaks, California: Sage Publications, 2014.

15. Manzano A. The craft of interviewing in realist evaluation. Evaluation 2016;22:342-60.

16. Mann S. Realist evaluation of the implementation of postpartum interventions in four African countries MOMI. London: University College London, 2015.

17. Jackson SF, Kolla G. A new realistic evaluation analysis method. $A m$ J Eval 2012;33:339-49.

18. Merton RK. Social theory and social structure. New York: The free press, 1968

19. Van Belle S, van de Pas R, Marchal B. Towards an agenda for implementation science in global health: there is nothing more practical than good (social science) theories. BMJ Glob Health 2017:2:1-4.

20. Westhorp G. Using complexity-consistent theory for evaluating complex systems. Evaluation 2012;18:405-20.

21. Hurst TE, Semrau K, Patna M, et al. Demand-side interventions for maternal care: evidence of more use, not better outcomes. BMC Pregnancy Childbirth 2015;15:297.
22. Strachan DL, Källander K, Nakirunda M, et al. Using theory and formative research to design interventions to improve community health worker motivation, retention and performance in Mozambique and Uganda. Hum Resour Health 2015:13:1-13.

23. Kane SS, Gerretsen B, Scherpbier R, et al. A realist synthesis of randomised control trials involving use of community health workers for delivering child health interventions in low and middle income countries. BMC Health Serv Res 2010;10:286.

24. Greenspan JA, McMahon SA, Chebet JJ, et al. Sources of community health worker motivation: a qualitative study in Morogoro Region, Tanzania. Hum Resour Health 2013;11:52.

25. Story WT. Social capital and health in the least developed countries: a critical review of the literature and implications for a future research agenda. Glob Public Health 2013;8:983-99.

26. McTavish S, Moore S. On est ensemble: social capital and maternal health care use in rural Cameroon. Global Health 2015;11:33.

27. Goss K. Putnam R, Democracies in flux the evolution of social capital in contemporary society. Oxford: Oxford University Press, 2002:1-19.

28. Rowe AK, de Savigny D, Lanata CF, et al. How can we achieve and maintain high-quality performance of health workers in low-resource settings? Lancet 2005;366:1026-35.

29. Franco LM, Bennett $S$, Kanfer R, et al. Health sector reform and public sector health worker motivation: a conceptual framework. Soc Sci Med 2002;54:1255-66.

30. Hulton L, Matthews Z, Martin-Hilber A, et al. Using evidence to drive action: a 'revolution in accountability' to implement quality care for better maternal and newborn health in Africa. Int $J$ Gynaecol Obstet 2014;127:96-101.

31. Wallace A, Dietz V, Cairns KL. Integration of immunization services with other health interventions in the developing world: what works and why? Systematic literature review. Trop Med Int Health 2009;14:11-19.

32. Dudley L, Garner P. Strategies for integrating primary health services in low- and middle-income countries at the point of delivery. Cochrane Database Syst Rev 2011;7:CD003318.

33. Nambiar B, Hargreaves DS, Morroni C, et al. Improving healthcare quality in resource-poor settings. Bull World Health Organ 2017;95:76-8. 